\title{
Generalización en las interacciones entre plantas y polinizadores
}

\author{
Generalizations in the interactions between plants and pollinators
}

\author{
JOSÉ M. GÓMEZ \\ Grupo de Ecología Terrestre, Departamento de Biología Animal y Ecología, \\ Facultad de Ciencias, Universidad de Granada, E-18071 Granada, España; \\ e-mail: jmgreyes@goliat.ugr.es
}

\begin{abstract}
RESUMEN
Hasta fechas muy recientes, la mayoría de los estudios teóricos y empíricos sobre la evolución de la relación entre las plantas y sus polinizadores han asumido que estos sistemas están altamente especializados, es decir, cada especie vegetal es visitada por sólo unos cuantos polinizadores relacionados filogenéticamente como fruto de la selección impuesta por ellos. La omnipresencia de especialización se ha derivado de aceptar el principio del polinizador más eficiente, según el cual una planta debe especializarse en el polinizador más eficaz y/o más abundante cuando su disponibilidad es predecible. Muchos estudios han encontrado realmente que los polinizadores ejercen selección fenotípica sobre rasgos reproductivos de las plantas. Sin embargo, igual de evidente es el hecho de que muchas especies vegetales son polinizadas por conjuntos numerosos y taxonómicamente diversos de polinizadores. Estas observaciones sugieren que en realidad los sistemas de polinización son generalistas. La generalización es producida y mantenida por varios factores ecológicos entre los que destacan la variación espacial y la fluctuación temporal de la identidad de los polinizadores más importantes, la similitud en la eficiencia y preferencia por los rasgos florales por parte de polinizadores diferentes, la existencia de factores externos que reducen la capacidad de los polinizadores de afectar al fitness de la planta, y la existencia de selección indirecta.
\end{abstract}

Palabras clave: generalización, especialización, ecología evolutiva, polinización, interacciones planta-polinizador.

\begin{abstract}
A controversy exists about the importance of generalization versus specialization in pollination systems. Whereas the evolutionary and ecological theory speculates that the plant-pollinator systems should be highly specialized, many evolutionary ecologists now acknowledge that generalization is frequent in natural systems. There is still no consensus about which ecological factors can promote this counter-intuitive and paradoxical result. I propose that generalization in plant-pollination systems can arise by several non-exclusive factors. One kind of factors are related to the ability of pollinators of acting as true selective agents. Despite that many studies have shown that pollinators can provoke phenotypic selection on floral traits, only occasionally it has been shown that this selection actually produces any response to selection. Two main reasons can explain why pollinators cannot induce adaptations in many plants: (1a) a significant spatial and temporal variability in pollinator abundance and identity highly reduces the possibilities of congruent selection on floral traits; (1b) the occurrence of extrinsic factors acting during another stages of the plant reproductive and recruitment processes can also decrease the actual effect that pollinators has on fitness. A second group of factors are those related with the fact that, to specialization occurs, it is necessary that two different pollinators can constitute as completely separated selective agents, by differing in their per-visit efficiencies and/or floral trait preferences. By contrast, similarity in efficiency and preference will constrain specialization even although pollinators act as selective pressures, since plant will have not any criteria to benefit a given pollinator species with respect to the other.
\end{abstract}

Key words: generalization, specialization, pollination biology, evolutionary ecology, plant-pollinator interactions.

\section{INTRODUCCIÓN}

Las complejas interacciones que se establecen entre las plantas y los animales que visitan sus flores han atraído el interés de los naturalistas desde hace más de dos siglos. La ecología de la polinización ha sido profusamente utilizada para apoyar el papel de la selección natural como motor evolutivo desde los primeros momentos de su formulación, como revelan los trabajos pioneros de Darwin $(1862,1877,1878)$ y muchos de sus coetáneos (Kerner 1878, Müller 1883, Kerner \& Oliver 1895, Knuth 1898-1905, Clements \& Long 1923). Desde entonces, los estudios sobre polinización han ido acumulando una tremenda cantidad de ejemplos de supuestas complejas adaptaciones y relaciones altamente evolucionadas entre insectos y plantas (Faegri \& van der Pijl 
1979, Barth 1989, Thompson 1994, Proctor et al. 1996). Por ello, el papel de los polinizadores como agentes selectivos de muchos caracteres reproductivos de las plantas está en la actualidad universalmente aceptada por la comunidad científica. De hecho, las interacciones entre las plantas y sus polinizadores han llegado a ser uno de los principales sistemas modelo donde estudiar la evolución adaptativa en la naturaleza (Campbell 1991, Johnston 1991, Campbell et al. 1997).

La mayoría de los estudios sobre la ecología evolutiva de las interacciones entre plantas y polinizadores se han gestado al amparo del denominado "principio del polinizador más eficiente" (Herrera 1996, Armbruster et al. 2000, Johnson \& Steiner 2000). Este principio, formulado originalmente por Stebbins (1970), postula que la selección natural favorecerá aquellos rasgos morfológicos, fisiológicos o ecológicos de las plantas que sirvan para atraer a aquellos visitantes florales que polinizan más eficientemente (Faegri \& van der Pijl 1979, Armbruster et al. 2000, Johnson \& Steiner 2000). Consecuentemente, según este principio la mayoría de las plantas deberían estar polinizadas por un grupo reducido de especies eficientes, fenómeno que se denomina especialización adaptativa mediada por polinizadores (Thompson 1994, Armbruster et al. 2000, Johnson \& Steiner 2000). Dándole el tiempo suficiente, la mayoría de los sistemas de polinización deberían tender hacia la especialización (Ollerton 1999, Johnson \& Steiner 2000). Así, algunas ideas que estructuran el estudio de las relaciones entre plantas y polinizadores son por ejemplo que la coevolución requiere especialización (Thompson 1994), que los sistemas de polinización evolucionan rápidamente en respuesta a presiones selectivas producidas por los polinizadores (Ollerton 1996), o que existe una tendencia histórica de especialización de tal forma que las plantas más "avanzadas" están más especializadas que las plantas más "primitivas" (Ollerton 1999).

Polinizadores relacionados filogenéticamente supuestamente ejercerán presiones selectivas similares, ya que exhibirán comportamiento y patrones de preferencias semejantes. Es de esperar por tanto que flores polinizadas por insectos similares también compartan rasgos semejantes. Este razonamiento permitió el surgimiento del concepto de síndrome de polinización, definido como el conjunto de rasgos florales que representan adaptaciones a tipos particulares de polinizadores (Faegri \& van der Pijl 1979), y que se extendió rápidamente entre los biólogos de la polinización por su aparente capacidad predictiva (Faegri \& van der Pijl 1979, Kampny 1995, Proctor et al. 1996). Mediante el estudio de los rasgos florales se puede descubrir cuales son los principales polinizadores de una determinada especies vegetal, y viceversa. La esencia del síndrome de polinización es, en resumen, la asociación de los caracteres florales entre sí, y de ellos con los polinizadores principales (Thomson et al. 2000).

Sin embargo, los datos de campo muestran que los sistemas de polinización generalistas son más frecuente de lo que los presupuestos teóricos dictaminan, ya que las flores de muchas especies vegetales son visitadas por un grupo numeroso y taxonómicamente diverso de insectos (e.g., Herrera 1988, Horvitz \& Schemske 1990, Gómez et al. 1996, Gómez \& Zamora 1999, Dilley et al. 2000, Lippok et al. 2000, Olesen 2000 y referencias allí, Thompson 2001), y en muchos casos, no existe una correlación positiva entre abundancia y eficiencia de los polinizadores de una determinada especies vegetal (Waser \& Price 1990, Vaughton 1992, Gómez \& Zamora 1999, Potts et al. 2001). En parte, esta realidad ha pasado desapercibida debido a que tradicionalmente los ecológos de la polinización han prestado atención sobretodo a aquellas especies de visitantes florales que son más adecuadas a priori para actuar como polinizador de la especie vegetal de estudio. De hecho, el número de sistemas de polinización generalistas estudiados hasta la fecha es aún escasa, lo que ha servido para inflar artificialmente la frecuencia de estudios sobre sistemas especializados de polinización (Waser et al. 1996, Gómez \& Zamora 1999).

En el presente artículo voy a repasar los requisitos necesarios para que ocurra especialización, comentando algunas posibles causas ecológicas que pueden propiciar la aparición y/o mantenimiento de sistemas de polinización generalistas. Algunos de estos factores ecológicos han sido ya apuntados en algunas revisiones previas sobre generalización en polinización, y remito al lector a que consulte dichos trabajos (Herrera 1996, Waser et al. 1996, Armbruster et al. 2000), mientras que otros de los factores que propongo nunca han sido considerados en los estudios sobre la evolución de la generalización. En cualquier caso no pretendo que la lista de causas ecológicas sea exhaustiva, ya que este ámbito de la ecología de polinización está aún en sus inicios y muchos otros factores se irán incorporando a medida que los estudios sobre polinización empiecen a tener en cuenta la complejidad del marco natural donde se desenvuelven las interacciones entre plantas y animales polinizadores. Asimismo, me gustaría dejar claro dos cuestiones previas. En primer lugar, no voy a revisar las posibles limitaciones genéticas o filogenéticas que también juegan a 
veces un papel importante en la existencia de sistemas de polinización generalizados. En segundo lugar, consideraré la generalización y la especialización desde una perspectiva tipológica, como dos soluciones opuestas y completamente disjuntas. Soy consciente de que esto supone una simplificación excesiva del problema, a pesar de lo cual he decidido adoptarla en aras de la didáctica, ya que considero que no afectará a la idea desarrollada en este trabajo (los lectores interesados en un tratamiento más exhaustivo y exacto del problema pueden consultar Olesen 2000).

\section{EFICIENCIA, FRECUENCIA DE INTERACCIÓN, E IMPORTANCIA DE UN POLINIZADOR}

Un concepto clave para poder analizar el debate en torno a las posibilidades de especialización o generalización en los sistemas de polinización es el de eficiencia de un polinizador. Desgraciadamente, aún no hay un consenso unánime y universal sobre qué entendemos por eficiencia de un polinizador. La mayoría de los investigadores la definen como el efecto que tiene dicho polinizador sobre el fitness de la planta por cada visita que efectúa (Herrera 1987, Fishbein \& Venable 1996, Waser et al. 1996, Gómez 2000a). Este concepto está pues relacionado con la calidad de un visitante floral como polinizador, y suele medirse en el número de granos de polen depositados o transportados, la proporción de cruzamientos alógamos realizados, la distancia de vuelo, el número de flores visitadas por planta consecutivamente, y muchas otras variables (Herrera 1987, Fishbein \& Venable 1996, Gómez \& Zamora 1999, Gómez 2000a, Potts et al. 2001).

No obstante, una estimación rigurosa del efecto de un polinizador en el fitness de la planta tiene que contemplar no sólo la eficiencia por visita sino también el número de visitas efectuadas por dicho polinizador. Este segundo aspecto está íntimamente relacionado con el componente cuantitativo de la interacción, y se puede denominar frecuencia de interacción. Se mide usualmente en parámetros poblacionales, como el número de flores visitadas por unidad de tiempo por una población de polinizador concreto (NFV según Herrera 1989). NFV tiene a su vez un subcomponente individual, número de flores visitadas por minuto por un polinizador concreto, $\mathrm{y}$ un subcomponente poblacional, número de polinizadores de esa especie visitando las flores por unidad de tiempo.

Integrando los dos componentes anteriores, el cualitativo y el cuantitativo podemos estimar la importancia de un polinizador para la planta, definida como el efecto de la población de dicho polinizador sobre el fitness de la planta (Olsen 1997). Desde mi punto de vista, este es el parámetro que nos ofrece una visión más completa de cual es la contribución real de un determinado polinizador al fitness de la planta.

La importancia de un polinizador es una función multiplicativa de su eficiencia por visita y su frecuencia de interacción. Ambos subcomponentes se pueden diferenciar a su vez en tantos parámetros como sean necesarios en cada sistema de estudio. Así, Stone (1996) estimó la importancia de los diferentes polinizadores del taxón neotropical Psychotria suerrensis (Rubiaceae) como $f \mathrm{x} e \mathrm{x} d$ $\mathrm{x} t \mathrm{x} q$, donde $f$ es la frecuencia relativa de visitas de cada taxón, $e$ es el número de plantas distintas visitadas por número de visitas, $d$ es la proporción de visitas diurnas antes de que el polen sea consumido completamente, $t$ es la cantidad de granos de polen transferido por visitas, y q es la proporción del total de granos de polen que eran compatible (donde $f, e, d$ y $q$ varían entre 0 y 1 ).

\section{PROCESO DE ESPECIALIZACIÓN}

El escenario de Stebbins presupone que la planta desarrollará los rasgos fenotípicos útiles para incrementar la frecuencia de interacción con el polinizador más eficiente. Varios requisitos son necesarios para que ocurra un proceso de especialización hacia el polinizador más eficiente. Yo los he diferenciado en dos grandes grupos que a su vez se diferencian en dos subgrupos cada uno.

\section{Los polinizadores deben constituirse como agen-} tes selectivos reales

Para que una planta desarrolle un rasgo que atraiga al polinizador más eficiente, un rasgo que sería considerado una adaptación, tiene que ocurrir un proceso de selección natural mediado por dicho polinizador. El proceso ocurre aproximadamente como se indica en la Fig. 1. Según este esquema, para que un polinizador actúe como agente selectivo: (1a) debe afectar al "fitness" de la planta. Debe existir una correlación significativa entre frecuencia de interacción con el polinizador y número de descendientes dejado por la planta. El problema es que la inmensa mayoría de los estudios realizados hasta la fecha utilizan estimadores excesivamente incompletos del fitness de la planta, como son por ejemplo la movilización de polen o la producción de semillas antes de la dispersión. Pero desde que la semilla se produce hasta que alcanza la edad de reproducción debe 
pasar por muchos estadíos vitales pre- y postdispersivos en los cuales el efecto del polinizador puede diluirse.

(1b) Debe provocar una relación positiva entre el valor de un rasgo determinado y el "fitness" de la planta, propiciando la existencia de selección fenotípica. Esto ocurre si el polinizador discrimina entre individuos de la misma especie vegetal basándose en uno o más caracteres fenotípicos de la flor. Para ello, debe existir una correlación positiva entre frecuencia de interacción y el valor del rasgo floral que va a ser seleccionado.

Cada polinizador debe constituirse como una unidad selectiva perfectamente diferenciable

Si una planta tiene que beneficiar a un polinizador en perjuicio de otros diferentes, un requisito indispensable es que exista algún mecanismo que permita a la planta diferenciar entre distintos visitantes florales. Los polinizadores deben ser unidades selectivas perfectamente diferenciables, lo que posibilita a la planta la generación de respuestas a la selección para atraer a los polinizadores más eficientes. Para que esto ocurra, también es necesario que se den dos pasos:

(2a) En primer lugar diferentes polinizadores deben tener diferente importancia o efecto sobre el "fitness" de la planta. Esto puede ocurrir bien porque los polinizadores tengan diferente abundancia o bien porque tengan diferente eficiencia con la misma abundancia (Waser et al. 1996). Por ejemplo, como reza el título de un artículo clásico, una condición necesaria para la especialización es la diferencia entre polinizadores en eficiencia (Schemske \& Horvitz 1984). El establecimiento de una jerarquía de polinizadores según sus importancias permitirá a la planta interpretar qué polinizador debe seleccionar (Schemske \& Horvitz 1984, Stanton et al. 1991, Conner et al. 1995, Galen 1999, Zamora 2000).

(2b) Asimismo, también debe ocurrir diferencia entre polinizadores en preferencia, ya que de esta un rasgo vegetal se podrá modificar para atraer a un determinado polinizador importante (Schemske \& Bradshaw 1999). Este mecanismo es el que posibilita que la frecuencia de interacción entre un polinizador eficiente y la planta aumente. Diferente preferencia floral por parte de los polinizadores previene cruzamientos indeseados (Waser 1998).

\section{FACTORES ECOLÓGICOS QUE FAVORECEN LA GENERALIZACIÓN EN LOS SISTEMAS DE POLINIZACIÓN}

Si alguna de las condiciones comentadas arriba no se cumplen, las probabilidades de que se produzca especialización hacia el polinizador más eficiente quedan mermadas. En esta sección voy a comentar las principales razones ecológicas que promueven o dificultan la especialización y posibilitan la generalización.

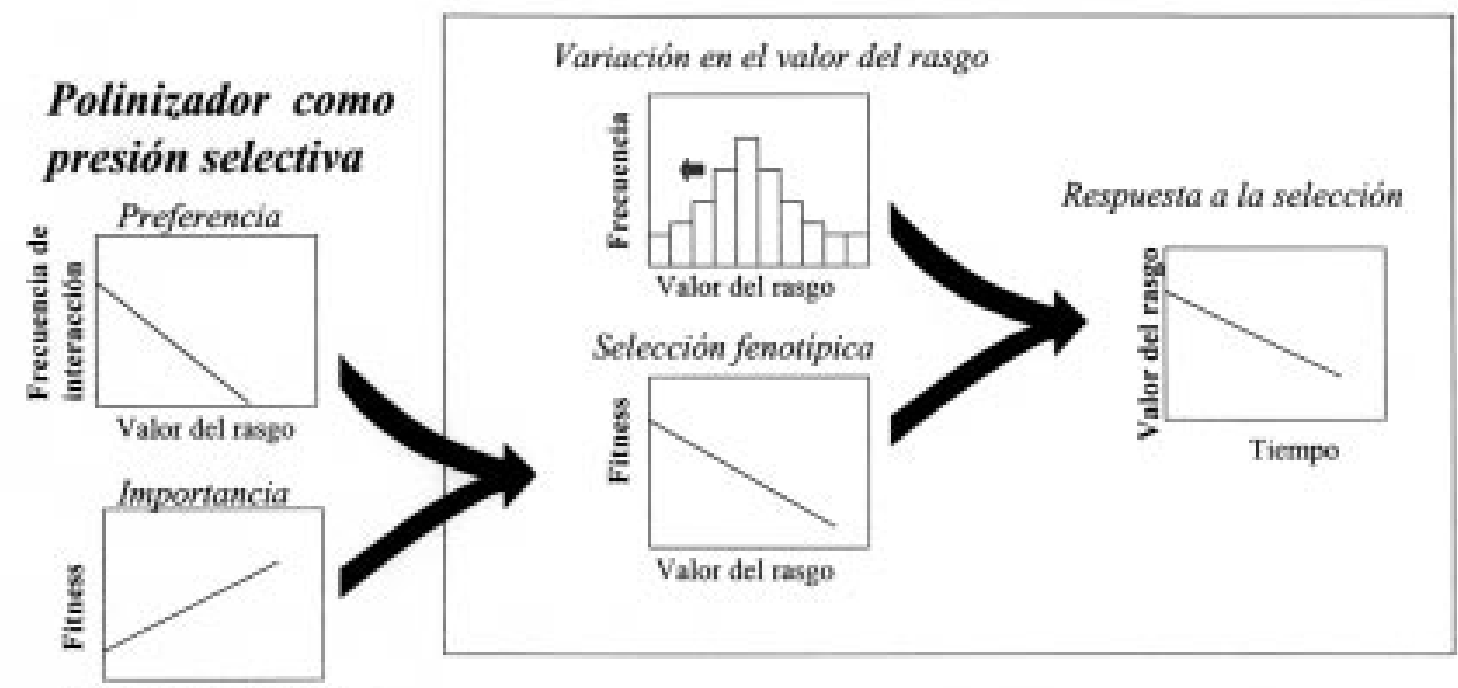

Frecuencia de inseracción

Fig. 1: Representación esquemática del proceso de selección natural mediado por polinizadores. Se indica los dos pasos o requisitos necesarios para que ocurre adaptación a un determinado polinizador.

A scheme of natural selection mediated through polinizers. Steps and requirements in order for adaptation to a particular polinizer are indicated. 
Fluctuaciones temporales en la abundancia de los visitantes florales

La fluctuación temporal en la abundancia y composición del conjunto de visitantes florales es una de las causas más aceptada de la existencia de sistemas de polinización generalizados. Una variación temporal en la identidad de los polinizadores más importantes para una especie vegetal puede provocar una concomitante fluctuación temporal en la dirección y magnitud de la selección fenotípica (Kalisz 1986, Schemske \& Horvitz 1989, Widén 1991, Andersson 1992, Domínguez \& Dirzo 1995, Maad 2000). Esto provoca la aparición de una incongruencia en las presiones selectivas soportadas por esa planta, y una dilución del proceso selectivo global ejercido por los polinizadores, lo que reduce las posibilidades de especialización al polinizador más efectivo (Herrera 1988, 1996, Waser et al. 1996).

Aunque las consecuencias del estilo de vida de la planta, si anual o perenne, en la relación entre fluctuación temporal y generalización aún no han sido analizadas en profundidad, es de esperar que no afecten a la idea central. La fluctuación temporal del gremio de polinizadores de una planta de vida larga provocará que la eficacia biológica relativa de un individuo determinado cambie entre años. De forma similar, en especies anuales lo que cambiará entre años será el número de individuos reclutados dependiendo de los caracteres morfológicos de los padres. En ambos casos, las consecuencias son las mismas, un determinado caracter fenotípico se beneficia unos años y otros no, dependiendo de su efecto sobre los polinizadores.

Un análisis riguroso de la variación temporal en el grupo de polinizadores demanda estudios a largo plazo. Los escasos estudios realizados usando serie temporales mayores de dos años han encontrado variación temporal en la abundancia, identidad e importancia de los polinizadores de la mayoría de las plantas estudiadas, como por ejemplo Lavandula latifolia (Herrera 1988), Silene vulgaris (Petterson 1991), Hormathophylla spinosa (Gómez \& Zamora 1992, 1999), Banksia spinulosa (Vaughton 1992), Asphodelus albus (Obeso 1992), Euphorbia dendroides (Traveset \& Sáez 1997), Campanula rotundifolia (Bingham 1998), Silene virginica (Fenster \& Dudash 2001) y Jasminum fruticans (Thompson 2001).

La composición y abundancia del conjunto de polinizadores puede variar incluso de forma estacional, dentro de un único periodo reproductivo de la planta. Por ejemplo, los principales polinizadores otoño-invernales de Lobularia maritima (Cruciferae) en áreas costeras del suroeste de la Península Ibérica son moscas, mientras que en verano son hormigas de la especie Camponotus micans (Gómez 2000a, 2000b).

Variación espacial en la abundancia de los visitantes florales

La variación espacial en la abundancia y composición del conjunto de visitantes florales es, junto con el factor anterior, el más usado para explicar la evolución de los sistemas de polinización generalistas. La argumentación que se sigue es la siguiente: La selección natural es un proceso eminentemente intrapoblacional. Por este motivo, si diferentes poblaciones de plantas están sometidas a diferentes escenarios selectivos, las presiones selectivas que reciben son diferentes, y esto podría generar incongruencia en las presiones selectivas globales actuando sobre la especie vegetal.

Algunos estudios realizados a una escala espacial suficientemente amplia están empezando a recoger datos sobre fluctuaciones significativa en la abundancia e identidad de los polinizadores más importantes de diversas especies de plantas (Herrera 1988, Horvitz \& Schemske 1990, Eckhart 1991, 1992, Guitián et al. 1996, Gómez \& Zamora 1999, Thompson 2001). Las causas que generan esta variación interpoblacional en el conjunto de polinizadores de una determinada especie vegetal son variadas, aunque se pueden agrupar en tres grupos: causas relacionadas con los requerimientos ecofisiológicos de los polinizadores (e.g., Herrera 1995, 1997), causas relacionadas con el tamaño poblacional de la especie vegetal en una determinada localidad (e.g., Eckhart 1991, Conner \& Neumeier 1995, Roll et al. 1997) y causas relacionadas con la presencia de otras especies vegetales con mayor poder atractor de visitantes florales (e.g., Caruso 2000).

Sin embargo, variación espacial, entre poblaciones, en la identidad de polinizadores, en ausencia de flujo génico efectivo entre poblaciones, podría producir una adaptación local a dicho conjunto de polinizadores. Cada población de plantas estaría sometida a diferentes escenarios selectivos. El resultado sería una planta generalista a nivel de especie que sin embargo es especialista a nivel poblacional, lo que formalmente se llama mosaico geográfico de la especialización (sensu Thompson 1994). Por este motivo, es muy importante determinar la existencia de dispersión vía polen o semilla entre poblaciones.

El conjunto de polinizadores puede variar incluso a escalas espaciales menores. A este respecto, algunos estudios han observado que diferentes 
individuos dentro de una población son preferentemente polinizados por distintos polinizadores dependiendo de su ubicación espacial en la población. Plantas de Lavandula latifolia cercanas a cursos de agua son polinizadas preferentemente por mariposas, mientras que plantas ubicadas en sitios más secos y soleados son polinizadas por abejas solitarias y las plantas situadas a la sombra son visitadas por dípteros (Herrera 1997). De forma similar, Zamora (1999) ha mostrado que las flores de individuos de Pinguicula vallisnerifolia ubicadas en paredes rocosas soleadas son visitadas preferentemente por abejas solitarias y el tisanóptero Taeniothrips meridionalis, mientras que las flores de plantas creciendo en las zonas no soleadas de la misma pared son visitadas sobre todo por el escarabajo Eusphalerum scribae (Staphylinidae). La existencia de esta estructuración espacial del conjunto de polinizadores es un factor importante a la hora de diluir las presiones selectivas globales, sobre todo si la dispersión via semilla no se estructura espacialmente de la misma forma.

Factores que diluyen el efecto de los polinizadores en el "fitness", o la importancia de considerar el ciclo vital completo de la planta

La mayoría de los estudios realizados hasta la fecha han estimado la eficiencia de los polinizadores, y su efecto en el "fitness" de la planta, cuantificando tan sólo el éxito reproductivo predispersivo de ésta, como por ejemplo la cantidad de granos de polen transportados o el número de semillas producidas por visita (Motten et al. 1981, Waser \& Price 1990, Dieringer 1992, Thompson \& Pellmyr 1992, Vaughton 1992, Keys et al. 1995, Stone 1996, Olsen 1997, Gómez \& Zamora 1999). Por el contrario, muy pocos estudios han tenido en cuenta si esos efecto predispersivos se mantienen durante otros estadíos importantes del proceso de reclutamiento de las especie vegetal, como la germinación de semillas, la emergencia de las plántulas y la supervivencia hasta adultos (ver Jennersten \& Morse 1991, Gómez 2000a, Herrera 2000a), a pesar de que estos parámetros demográficos ofrecen una estima mucho más realista y completa del efecto de los polinizadores en el fitness de la planta, y de la calidad de los eventos de polinización (Gómez 1996, Johnston \& Schoen 1996, Husband \& Schemske 1997). Así, algunas características de los polinizadores relacionadas con su eficiencia, como la frecuencia de polinizaciones geitonógamas, la distancia de vuelo promedio, la capacidad de arrastrar polen durante más de un visita ("pollen carryover"), etc., afectan a la depresión endogámica de la planta (Snow et al. 1996). Sin embargo, la endogamia es específica del estadío vital para muchas especies de plantas, y mientras plantas alógamas expresan la endogamia durante la fase de producción de semillas, las especies autógamas y geitonógamas tienden a expresarla en estadíos posteriores del proceso de reclutamiento de nuevos individuos (Husband \& Schemske 1996).

La importancia de tener en cuenta todo el proceso de reclutamiento de la planta estriba en el hecho de que, al constar éste de muchas fases concatenadas secuencialmente de forma multiplicativa, un colapso en una de las fases provoca un colapso general del proceso completo. Esto conlleva que un efecto intenso de los polinizadores en la producción de semillas pueda verse anulado por efectos ecológicos extrínsecos de signo contrario aconteciendo en estadíos posteriores del reclutamiento, disminuyendo también la variación en el fitness de la planta atribuible a ciertos caracteres seleccionados por los polinizadores (oportunidad para la selección). Esto sugiere que cualquier factor que anule el reclutamiento en una fase de éste posterior a la polinización diluirá el efecto del polinizador sobre el fitness, y reducirá la capacidad de la planta de responder a la selección fenotípica impuesta por éste.

Factores ecológicos extrínsecos que afectan al reclutamiento de las plantas pueden ser bióticos, como los herbívoros, o abióticos, como el clima. Por ejemplo, en zonas áridas del sudeste ibérico las flores de Moricandia moricandioides están sometidas a selección fenotípica por parte de sus polinizadores más importantes, varias especies de ábejas antofóridas, si usamos como componente del fitness la producción de semillas. Sin embargo, en las condiciones actuales las plantas no pueden responder a esta selección ya que la mayoría de estas semillas producidas son posteriormente consumidas por los ungulados domésticos (Gómez 1996). De hecho, analizando como componentes del fitness aquellos localizados en fases posteriores del proceso de reclutamiento, como por ejemplo la cantidad de semillas dispersadas exitosamente, encontramos que factores externos al sistema, como por ejemplo el microhábitat donde crece la planta, explican más variabilidad entre plantas en fitness que la morfología floral (Gómez 1996). De forma similar, los ungulados son los principales herbívoros de Hormathophylla spinosa en Sierra Nevada (España), ya que son capaces de reducir la fecundidad de las plantas hasta un $70 \%$, y pueden explicar más del $50 \%$ de la variación entre plantas en el 
componente femenino del fitness (Gómez 1993, Gómez \& Zamora 2000). Por todo esto, en aquellas poblaciones de plantas donde los ungulados están presentes, la variabilidad en fitness explicada por los polinizadores es tan solo del 0,05\%, lo cual diluye bastante su capacidad de actuar como presiones selectivas. Además, en algunas poblaciones los ungulados tienen criterios de preferencias similares a los de los polinizadores, alimentándose preferentemente de las plantas que producen muchas flores. Esto produce un conflicto entre los regímenes de selección ejercidos por organismos antagónicos y mutualistas, disminuyendo aún más el papel de los polinizadores como presiones selectivas (Gómez \& Zamora 2000). Herrera (2000b) ha llegado a una conclusión similar estudiando el efecto de los polinizadores sobre Paeonia broteroi (Paeoniaceae) en la Sierra de Cazorla (España), donde la oportunidad de adaptación de la planta a los polinizadores está fuertemente limitada por el intenso daño provocado por ungulados silvestres.

Similar efectividad por parte de polinizadores distintos

Según los presupuestos teóricos, hay oportunidad para especialización sólo si diferentes visitantes florales tienen diferente efectividad como polinizadores (Schemske \& Horvitz 1984, 1989, Herrera 1987, 1989, Eckhart 1991, 1992, Stanton et al. 1991, Fishbein \& Venable 1996, Zamora 2000). Por el contrario, cuando diferentes especies de visitantes florales poseen similar eficiencia como polinizadores, no existe a-priori ningún criterio para favorecer la interacción con alguna de ellas en particular. El resultado es un sistema de polinización generalistas en el que más de una especie animal actúa como polinizador de la misma especie vegetal (Gómez \& Zamora 1999). Algunos estudios han encontrado de hecho que diferentes polinizadores parecen tener eficiencias similares (Petterson 1991, Dieringer 1992, Vaughton 1992, Thøstesen \& Olesen 1996, Gómez \& Zamora 1999, Gómez 2000a).

Las razones que favorecen similitud entre polinizadores son variadas. Una flor simple de morfología poco restrictiva puede provocar que diferentes especies depositen la misma cantidad de granos de polen por visita independientemente de sus características morfológicas o hábitos tróficos (nectarívoro o polinívoro). En este caso, existe un bucle retroalimentativo de tal forma que una flor generalizada tenderá a seguir siéndolo. No obstante, diferentes polinizadores pueden ser igual de eficientes incluso polinizando una flor con morfología compleja y restrictiva. Así, Thøstesen \& Olesen (1996) encontraron que el abejorro especialista Bombus consobrinus tiene la misma eficiencia removiendo polen de Aconitum septentrionale que los abejorros generalistas $B$. jonellus, B. pratorum o B. wurfleinii. Cuando la flor es de morfología compleja, algunas carácterísticas ayudan a que diferentes polinizadores sean igual de eficientes. Así, es más probable que dos polinizadores sean igual de eficientes para una planta con pocos ovulos por flor, que necesita una carga de polen mínima para la fecundación de todos ellos y los granos de polen adicionales no juegan ningún papel o incluso entorpecen el proceso de fecundación, en comparación con una planta con muchos ovulos por flor (Johnson et al. 1995). O también puede haber similitud de eficiencias para aquellas especies de plantas en las que lo que importa es la procedencia del polen y no su cantidad, y su arquitectura favorezca que todos los polinizadores desarrollen comportamiento de pecoreo similar (Gómez \& Zamora 1999).

En este tipo de sistemas generalistas la importancia de un visitante floral como polinizador depende principalmente de su abundancia en las flores, o frecuencia de interacción (Gómez \& Zamora 1992, 1999, Vaughton 1992, Thøstesen \& Olesen 1996), más que de su eficiencia por visita, la cual es similar entre todos los polinizadores. Esto contradice la idea de que algunos sistemas aparentemente generalistas sean realmente sistemas funcionalmente especialistas, donde de todas las especies de insectos sólo una o dos sean responsables de la mayoría de las polinizaciones (Ollerton 1999). De hecho, en este tipo de sistemas generalistas la identidad de los polinizadores más importantes pueden cambiar entre años o sitios sin ningún efecto sobre los regímenes selectivos, ya que los polinizadores son completamente intercambiables.

Diferentes polinizadores tienen similar preferencia por los rasgos florales

Cuando los visitantes florales difieren en su beneficio para la planta, la selección natural modificará los rasgos florales con el objeto de atraer al polinizador más eficiente (Waser et al. 1996). Sin embargo, algo que pocas veces se ha tenido en cuenta es que este proceso sólo es posible si diferentes polinizadores difieren en sus preferencias por los rasgos florales (Waser et al. 1996, Wilson \& Thomson 1996, Campbell et al. 1997, Galen 1999, Schemske \& Bradshaw 1999). Si, por el contrario, diferentes polinizadores tienen 
similares patrones de preferencia con respecto a un determinado rasgo floral, la selección natural no podrá diferenciar entre ellos, y seleccionándose el más eficiente mediante la modificación de dicho rasgo también se seleccionarán los menos eficientes. Curiosamente, la variación entre polinizadores en sus patrones de preferencias hacia los rasgos vegetales ha llamado pocas veces la atención de los investigadores, a pesar de las gran importancia para la evolución vegetal que esto pueda tener (Johnson et al. 1995, Conner \& Rush 1996, Wilson \& Thomson 1996, Thompson 2001). Algunos estudios han mostrado que la preferencia de diferentes polinizadores, relacionados filogenéticamente o no, convergen en relación a los rasgos florales. Por ejemplo, similares patrones de preferencias han sido mostrados entre abejas solitarias y sírfidos con respecto al tamaño de las flores de Raphanus raphanistrum (Conner \& Rush 1996), entre abejas y mariposas para el tamaño de la flor de Wurmbea dioica (Vaughton \& Ramsey 1998), entre moscas y hormigas para el tamaño y número de flores de Lobularia maritima (Gómez 2000b), y entre algunas especies de abejorros con respecto a la exserción del estigma de Erythronium grandiflorum o la morfología floral completa de Impatiens pallida (Wilson \& Thomson 1996).

Una consecuencia que se deriva de lo explicado anteriormente es que en este tipo de sistemas es muy probable que todos los polinizadores ejerzan presiones selectivas similares sobre la planta (Wilson \& Thompson 1996).

Diferentes polinizadores seleccionan diferentes rasgos no correlacionados genéticamente

Finalmente, una razón adicional que puede favorecer la aparición y mantenimiento de sistemas generalistas de polinización ocurre cuando diferentes polinizadores prefieren (seleccionan) diferentes rasgos vegetales no correlacionados genéticamente. La mayoría de los estudios sobre el papel de los polinizadores como presiones selectivas se han centrado en los efectos sobre un único rasgo o sobre varios rasgos pero de forma independiente. Sin embargo, el fenotipo de cualquier organismo está compuesto por multitud de rasgos. Un sistema de polinización puede permanecer generalizado cuando cada polinizador o grupo de polinizadores seleccionan ciertos rasgos que no afectan a la relación entre la planta y otros polinizadores diferentes. En esta línea, Dilley et al. (2000) han descubierto que algunas innovaciones en las glándulas florales del género Calochortus (Liliaceae) favorece la visita por escarabajos sin dificultar la visita por abejas. Según estos autores, este tipo de adaptaciones no conflictivas ayudan al mantenimiento de sistemas de polinización generalizado (Dilley et al. 2000). Bajo este prisma, podemos considerar a una flor como un agregados de carácteres, cada uno de ellos atractivos para diferentes tipos de polinizadores. Si estos carácteres no están correlacionados entre sí, las presiones selectivas que ejerce cada tipo de polinizador podrían ser independientes. Sin embargo, no está claro cuán frecuente es este mecanismo, si tenemos en cuenta que las flores de la mayoría de las especies vegetales son órganos altamente integrados cuyos rasgos individuales están altamente correlacionados entre sí (e.g., Armbruster et al. 1999).

\section{¿SON GENERALISTAS LOS POLINIZADORES?}

Aunque igual de importante para el estudio de la coadaptación y coevolución entre plantas y polinizadores, mucho menos esfuerzo se ha dedicado a explorar el grado de especialización que muestran los visitantes florales. Los pocos trabajos esporádicos que hay sobre el tema usualmente muestran que la generalización se da en los visitantes florales si cabe de forma más acusada que en las plantas (Memmott 1999). Sánchez Piñero (1994) analizó durante dos años las preferencias tróficas de 66 especies de coleópteros antófilos de una zona semiárida del sureste de la Península Ibérica, y encontró que la mayoría de ellos se alimentaban de las flores de al menos 10 especies diferentes de plantas no relacionadas taxonómica y morfológicamente, y que existía una fuerte variación espacio-temporal en las preferencias tróficas de las principales especies de coleópteros. Petanidou \& Vokou (1993) encontraron que de 95 especies de abejas solitarias visitantes de las flores de 10 especies de labiadas en zonas de matorrales de Grecia, tan sólo 15 tenían un hábito trófico especialista (monotropo). De forma similar, el exhaustivo estudio de Struck (1994) sobre el comportamiento de más de 300 especies de insectos antófilos de zonas áridas de Sudafrica, y pertenecientes a 41 familias, claramente muestra que la generalización es la principal característica de la fauna.

Estos escasos estudios sugieren, por tanto, que el grado de especialización no es mucho mayor entre las especies de visitantes florales. En cualquier caso, sería deseable acumular más información sobre las interacciones mutuas, para poder vislumbrar la existencia de patrones de estructuración de las redes tróficas de las plantas y sus visitantes florales (Memmott 1999). 


\section{LA DIMENSIÓN GEOGRÁFICA DE LA GENERALIZACIÓN}

Un aspecto que aún no ha sido explorado en profundidad es la posibilidad de que exista una estructuración geográfica de la generalización. Ya hay suficientes estudios de campo como para poder revisar en qué tipo de ecosistemas es más probable la existencia de sistemas de polinización generalistas. Responder a esta pregunta tiene una doble importancia. Por una parte, permite sacar conclusiones del efecto del contexto ecológico en la estructura de las redes tróficas de polinizadores y flores. Por otro lado, puede arrojar luz sobre las razones por las cuales algunos autores piensan que la especialización es un resultado evolutivo común en las relaciones entre plantas y polinizadores mientras que otros autores suponen que el resultado será más parecido a la generalización.

El clima local y regional puede constreñir en mayor o menor medida las respuestas de las plantas a las presiones ejercidas por los polinizadores. Por ejemplo, parece que la mayoría de las plantas que habitan el Mediterráneo tienen sistemas de polinización altamente generalistas, independientemente de su morfología floral más o menos restrictiva (e.g., Herrera 1988, 1996, Gómez \& Zamora 1992, 1999, Obeso 1992, Petanidou \& Vokou 1993, Gómez et al. 1996, Guitián et al. 1996, Bosch et al. 1997, Traveset \& Saez 1997, Bosch et al. 1998, Gómez 2000a, Thompson 2001, Potts et al. 2001). Sería interesante explorar si la generalización es más frecuente en ambientes estacionales o intrínsecamente heterogéneos que en ambientes más homogéneos.

\section{CONSIDERACIONES FINALES}

La generalización en los sistemas de polinización ha sido tradicionalmente considerada una consecuencia de los factores ecológicos, genéticos o filogenéticos que limitan el desarrollo de sistemas especializados. Esta presunción ha provocado que la generalización haya sido considerada usualmente como un fenómeno no adaptativo. Generalización tan sólo ocurre cuando no puede existir especialización. Sin embargo, con este ensayo he intentado proponer que en aquellos escenarios ecológicos en los que los polinizadores son agentes selectivos reales pero tienen eficiencia y patrones de preferencia similares, la selección natural provocada por estos polinizadores puede promover sistemas de polinización generalistas. Estos sistemas en los que la morfología floral es fruto de la selección impuesta por un grupo de polinizadores taxonómicamente heterogéneo pero ecológica y selectivamente homogéneo, deberían ser considerado sistemas generalistas adaptativos" o generalización adaptativa, para contraponerlos a aquellos otros sistemas en los que la generalización acontece mayormente debido a la incapacidad de los polinizadores de configurarse como presiones selectivas, bien por la existencia de variabilidad espacio-temporal o por la acción de factores extrínsecos.

Independientemente de estas consideraciones sobre el papel de la selección natural, el reconocimiento de la existencia de sistemas de polinización generalistas es ya una realidad (Herrera 1996, Waser et al. 1996, Gómez \& Zamora 1999, Armbruster et al. 2000, Olesen 2000). Por ello, para terminar este trabajo, me gustaría proponer algunas cuestiones importantes relacionadas con la generalización que serían interesante comenzar a explorar: (1) cuán frecuente son los sistemas generalistas, y cuáles son los principales mecanismos que provocan dicha generalización; (2) existe variación geográfica y/o entre biomas en la frecuencia de los sistemas de polinización generalistas; (3) existe diferencia taxonómica o filogenética en el grado de generalización de las especies vegetales; (4) existe correlación entre complejidad morfológica floral y grado de especialización. Estoy convencido de que la resolución de estas cuestiones ayudaría a profundizar aún más en el conocimiento real y objetivo de la evolución de las interacciones entre las plantas y sus polinizadores.

\section{AGRADECIMIENTOS}

Agradezco a Anna Traveset y a Mary Kalin-Arroyo la invitación a participar en el Taller sobre "Aspectos Teóricos de la Reproducción en Plantas: Consecuencias para la Conservación”, organizado por el Programa Iberoamericano de Ciencia y Tecnología para el Desarrollo (CYTED), así como su hospitalidad en Palma de Mallorca (España). Agradezco también al Dr. C.M. Herrera y a un revisor anónimo sus acertados comentarios sobre una versión preliminar de este manuscrito.

\section{LITERATURA CITADA}

ANDERSSON R (1992) Phenotypic selection in a population of Crepis tectorum ssp. pumila (Asteraceae). Canadian Journal of Botany 70: 89-95. 
ARMBRUSTER WS, VS DI STILIO, JD TUXILL, TC FLORES \& JL VELÁSQUEZ-RUNK (1999) Covariance and decoupling of floral and vegetative traits in nine Neotropical plants: a re-evaluation of Berg's correlation-pleiades concept. American Journal of Botany 86: 39-55.

ARMBRUSTER WS, CB FENSTER \& MR DUDASH (2000) Pollination "principle" revisited: specialization, pollination syndromes, and the evolution of flowers. Det Norske VidenskapsAkademi. I. Matematisk Naturvidensskapelige Klasse, Skrifter, Ny Serie 39: 179-200.

BARTH FG (1989) Insects and flowers, the biology of a partnership. Princeton University Press, Princeton, New Jersey. 408 pp.

BINGHAM RA (1998) Efficient pollination of alpine plants. Nature 391: 238.

BOSCH J, J RETANA \& X CERDÁ (1997) Flowering phenology, floral traits and pollinator composition in a herbaceous Mediterranean plant community. Oecologia 109: 583-591.

BOSCH M, J SIMON, J MOLERO \& C BLANCHÉ (1998) Reproductive biology, genetic variation and conservation of the rare endemic dysploid Delphinium bolosii (Ranunculaceae). Biological Conservation 86 : 57-66.

CAMPBELL DR (1991) Measurement of selection in a hermaphroditic plant: variation in male and female pollination success. Evolution 43: 318-334.

CAMPBELL DR, NM WASER \& EJ MELÉNDEZACKERMAN (1997) Analyzing pollinator-mediated selection in a plant hybrid zone: hummingbird visitation patterns on three spatial scales. American Naturalist 149: 295-315.

CARUSO CM (2000) Competition for pollination influences selection on floral traits of Ipomopsis aggregata. Evolution 54: 1546-1557.

CLEMENTS FE \& FL LONG (1923) Experimental pollination, an outline of the ecology of flowers and insects. Carnegie Institution of Washington, Publication No. 336. 340 pp.

CONNER JK \& R NEUMEIER (1995) Effects of black mustard population size on the taxonomic composition of pollinators. Oecologia 104: 218-224.

CONNER JK \& S RUSH (1996) Effects of flower size and number on pollinator visitation to wild radish, Raphanus raphanistrum. Oecologia 105: 509-516.

CONNER JK, R DAVIS \& S RUSH (1995) The effect of wild radish floral morphology on pollination efficiency by four taxa of pollinators. Oecologia 104: 234-245.

DARWIN C (1862) The various contrivances by which British and foreign orchids are fertilisez by insects. Murray, London, United Kingdom. 300 pp.

DARWIN C (1877) The effects of cross and self fertilisation in the vegetable kingdom. Murray, London, United Kingdom. 352 pp.

DARWIN C (1878) The different forms of flowers on plants of the same species. Murray, London, United Kingdom. 352 pp.

DIERINGER G (1992) Pollinator effectiveness and seed set in populations of Agalinis strictifolia (Scrophulariaceae). American Journal of Botany 79: 1018-1023.
DILLEY JD, P WILSON \& MR MESLER (2000) The radiation of Calochortus: generalist flowers moving through a mosaic of potential pollinators. Oikos 89 : 209-222.

DOMÍNGUEZ CA \& R DIRZO (1995) Rainfall and flowering synchorny in a tropical shrub: variable selection on the flowering time of Erythroxylum havanense. Evolutionary Ecology 9: 204-216.

ECKHART VM (1991) The effects of floral display on pollinator visitation vary among populations of Phacelia linearis (Hydrophyllaceae). Evolutionary Ecology 5: 370-384.

ECKHART VM (1992) Spatio-temporal variation in abundance and variation in foraging behavior of the pollinators of gynodioecious Phacelia linearis (Hydrophyllaceae). Oikos 64: 573-586.

FAEGRI K \& L VAN DER PIJL (1979) The principles of pollination ecology. Tercera edición. Pergamon Press, Oxford, United Kingdom. 244 pp.

FENSTER CB \& MR DUDASH (2001) Spatiotemporal variation in the role of hummingbirds as pollinators of Silene virginica. Ecology 82: 844-851.

FISHBEIN M \& DL VENABLE (1996) Diversity and temporal change in the effective pollinators of Asclepias tuberosa. Ecology 77: 1061-1073.

GALEN C (1999) Why do flowers vary? Bioscience 49: 631-640.

GÓMEZ JM (1993) Phenotypic selection on flowering synchrony in a high mountain plant, Hormathophylla spinosa (Cruciferae). Journal of Ecology 81: 605613.

GÓMEZ JM (1996) Predispersal reproductive ecology of an arid land crucifer, Moricandia moricandioides: effect of mammal herbivory on seed production. Journal of Arid Environments 33: 425-437.

GÓMEZ JM (2000a) Effectiveness of ants as pollinators of Lobularia maritima: effects on main sequential fitness components of the host plant. Oecologia 122: 90-97.

GÓMEZ JM (2000b) Phenotypic selection and response to selection in Lobularia maritima: importance of direct and correlational components of natural selection. Journal of Evolutionary Biology 13: 689-699.

GÓMEZ JM \& R ZAMORA (1992) Pollination by ants: consequences of the quantitative effect on a mutualistic system. Oecologia 91:410.418.

GÓMEZ JM \& R ZAMORA (1999) Generalization vs. specialization in the pollination system of Hormathophylla spinosa (Cruciferae). Ecology 80: 796-805.

GÓMEZ JM \& R ZAMORA (2000) Spatial variation in the selective scenarios of Hormathophylla spinosa (Cruciferae). American Naturalist 155: 657-668.

GÓMEZ JM, R ZAMORA, JA HÓDAR \& D GARCÍA (1996) Experimental study of pollination by ants in Mediterranean high mountain and arid habitats. Oecologia 105: 236-242.

GUITIÁN J, P GUITIÁN \& L NAVARRO (1996) Spatiotemporal variation in the interactions between Cornus sanguinea and its pollinators. Acta Oecologica 17: 285-295.

HERRERA CM (1987) Components of pollinator «quality»: comparative analysis of a diverse insect assemblage. Oikos 50: 79-90. 
HERRERA CM (1988) Variation in mutualism: the spatiotemporal mosaic of a pollinator asemblage. Biological Journal of the Linnean Society 35: 95-125.

HERRERA CM (1989) Pollinator abundance, morphology, and flower visitation rate: analysis of the "quantity" component in a plant-pollinator system. Oecologia 80: 241-248.

HERRERA CM (1995) Microclimate and individual variation in pollinators: flowering plants are more than their flowers. Ecology 76: 1516-1524.

HERRERA CM (1996) Floral traits and plant adaptation to insect pollinators: a devil's advocate approach. En: Lloyd DG \& SCH Barrett (eds) Floral biology: 65-87. Chapman and Hall, New York, New York.

HERRERA CM (1997) Thermal biology and foraging responses of insect pollinators to the forest floor irradiance mosaic. Oikos 78: 601-611.

HERRERA CM (2000a) Flower-to-seedling consequences of different pollination regimes in an insect-pollinated shrub. Ecology 81: 15-29.

HERRERA CM (2000b) Measuring the effects of pollinators and herbivores: evidence for non-additivity in a perennial herb. Ecology 81: 2170-2176.

HOR VITZ CC \& DW SCHEMSKE (1990) Spatiotemporal variation in insect mutualists of a neotropical herb. Ecology 71: 1085-1097.

HUSBAND BC \& DW SCHEMSKE (1996) Evolution of the magnitude and timing of inbreeding depression in plants. Evolution 50: 54-70.

HUSBAND BC \& DW SCHEMSKE (1997) The effect of inbreeding in diploid and tetraploid populations of Epilobium angustifolium (Onagraceae): implications for the genetic basis of inbreeding depression. Evolution 51: 737-746.

JENNERSTEN O \& DH MORSE (1991) The quality of pollination by diurnal and nocturnal insects visiting common milkweed, Asclepias syriaca. American Midland Naturalist 125: 18-28.

JOHNSON SD \& KE STEINER (2000) Generalization versus specialization in plant pollination systems. Trends in Ecology and Evolution 15: 140-143.

JOHNSON SG, LF DELPH \& CL ELDERKIN (1995) The effect of petal-size manipulation on pollen removal, seed set, and insect-visitor behavior in Campanula americana. Oecologia 102: 174-179.

JOHNSTON MO (1991) Natural selection on floral traits in two species of Lobelia with different pollinators. Evolution 45: 1468-1479.

JOHNSTON MO \& DJ SCHOEN (1996) Correlated evolution of self-fertilization and inbreeding depression: an experimental study of nine populations of Amsinckia (Boraginaceae). Evolution 50: 14781491.

KALISZ S (1986) Variable selection on the timing of germination in Collinsia verna (Scrophulariceae). Evolution 40: 479-491.

KAMPNY CM (1995) Pollination and flower diversity in Scrohulariaceae. Botanical Review 61: 350-366.

KERNER A VON M (1878) Flowers and their unbidden guests. C. Kegan Paul \& Co., London, United Kingdom. 348 pp.
KERNER A VON M \& FW OLIVER (1895) The natural history of plants. Their form, growth, reproduction, and distribution. Blackie \& Sons, London, United Kingdom. 777 pp.

KEYS RN, SL BUCHMANN \& SE SMITH (1995) Pollination effectiveness and pollination efficiency of insects foraging Prosopis velutina in south-eastern Arizona. Journal of Applied Ecology 32: 519-527.

KNUTH P (1898-1905) Handbüch der Blutenbiologie. Volume I-III. W. Engelmann, Leipzig, Alemania. 400, 697 y 705 pp.

LIPPOK B, AA GARDINE, PS WILLIAMSON \& SS RENNER (2000) Pollination by flies, bees, and beetles of Nuphar ozarkana and N. advena (Nymphaeaceae). American Journal of Botany 87: 898-902.

MAAD J (2000) Phenotypic selection in hawkmothpollinated Platanthera bifolia: targets and fitness surfaces. Evolution 54: 112-123

MEMMOTT J (1999) The structure of a plant-pollinator food web. Ecology Letters 2: 276-280.

MOTTEN AF, DR CAMPBELL, DE ALEXANDER \& HL MILLER (1981) Pollination effectiveness of specialist and generalist visitors to a north Carolina population of Claytonia virginica. Ecology 62: 1278-1287.

MÜLLER H (1883) The fertilisation on flowers. MacMillan \& Company, New York, New York. 669 pp.

OBESO JR (1992) Pollination ecology and seed set in Asphodelus albus (Liliaceae) in northern Spain. Flora 187: 219-226.

OLESEN JM (2000) Exactly how generalised are pollination interactions? Det Norske VidenskapsAkademi. I. Matematisk Naturvidensskapelige Klasse, Skrifter, Ny Serie 39: 161-178.

OLLERTON J (1996) Reconciling ecological processes with phylogenetic patterns: the apparent paradox of plant-pollinator systems. Journal of Ecology 84: 767 769.

OLLERTON J (1999) La evolución de las relaciones polinizador-planta en los Artrópodos. Boletín de la Sociedad Entomológica Aragonesa (España) 26: 741 758.

OLSEN KM (1997) Pollinator effectiveness and pollinator importance in a population of Heterotheca subaxillaris (Asteraceae). Oecologia 109: 114-121.

PETANIDOU T \& D VOKOU (1993) Pollination ecology of Labiatae in a phryganic (East Mediterranean) ecosystem. American Journal of Botany 80: 892-899.

PETTERSON MW (1991) Pollination by a guild of fluctuating moth populations: option for unspecialization in Silene vulgaris. Journal of Ecology 79: 591-604.

POTTS SG, A DAFNI \& G NE'EMAN (2001) Pollination of a core flowering shrub species in Mediterranean phrygana: variation in pollinator diversity, abundance and effectiveness in response to fire. Oikos 92: 7180.

PROCTOR M, P YEO \& A LACK (1996) The natural history of pollination. Timber Press, Portland, Oregon. $479 \mathrm{pp}$.

ROLL J, RJ MITCHELL, RJ CABIN \& DL MARSHALL (1997) Reproductive success increases with local density of conspecific in a desert mustard (Lesquerella fendleri). Conservation Biology 11: 738-746 
SÁNCHEZ-PIÑERO F (1994) Ecología de las comunidades de coleópteros en zonas áridas de la depresión de Guadix-Baza (Sureste de la Península Ibérica). Tesis Doctoral inédita, Universidad de Granada, Granada, España. 313 pp.

SCHEMSKE DW \& HD BRADSHAW (1999) Pollinator preference and the evolution of floral traits in monkeyflowers (Mimulus). Proceedings of the National Academy of Sciences USA 96: 11910-11915.

SCHEMSKE DW \& CC HORVITZ (1984) Variation among floral visitors in pollination ability: a precondition for mutualism specialization. Science 225: 519-521.

SCHEMSKE DW \& CC HORVITZ (1989) Temporal variation in selection on a floral character. Evolution 43: 461-465.

SNOW AA, TP SPIRA, R SIMPSON \& RA KLIPS (1996) The ecology of geitonogamous pollination. En: Lloyd DG \& SCH Barrett (eds) Floral biology: 191-216. Chapman and Hall, New York, New York.

STANTON M, HJ YOUNG, NC ELLSTRAND \& JM CLEGG (1991) Consequences of floral variation for male and female reproduction in experimental populations of wild radish, Raphanus sativus L. Evolution 45: 268-280.

STEBBINS GL (1970) Adaptive radiation of reproductive characteristics in Angiosperms, I: pollination mechanisms. Annual Review of Ecology and Systematics 1: 307-326.

STONE JL (1996) Components of pollination effectiveness in Psychotria suerrensis, a tropical distylous shrub. Oecologia 107: 504-512.

STRUCK M (1994) Flowers and their insect visitors in the arid winter rainfall region of southern Africa: observations on permanent plots. Insect visitation behaviour. Journal of Arid Environments 28: 51-74.

THOMPSON JD (2001) How do visitation patterns vary among pollinators in rlation to floral display and floral desing in a generalist pollination system? Oecologia 126: 386-394.

THOMPSON JN (1994) The coevolutionary process. University Press of Chicago Press, Chicago, Illinois. $376 \mathrm{pp}$.

THOMPSON JN \& O PELLMYR (1992) Mutualism with pollinating seed parasites amid co-pollinators: constraints on specialization. Ecology 73: 1780-1791.
THOMSON JD, P WILSON, M VALENZUELA \& M MALZONE (2000) Pollen presentation and pollination syndromes, with special reference to Penstemon. Plant Species Biology 15: 11-29.

THØSTESEN AM \& JM OLESEN (1996) Pollen removal and deposition by specialist and generalist bumblebees in Aconitum septentrionale. Oikos 77: 77-84.

TRAVESET A \& E SÁEZ (1997) Pollination of Euphorbia dendroides by lizards and insects: spatio-temporal variation in patterns of flower visitation. Oecologia 111: 241-248.

VAUGHTON G (1992) Effectiveness of nectarivorous birds and honeybees as pollinators of Banksia spinulosa (Proteaceae). Australian Journal of Ecology 17: 43-50.

VAUGHTON G \& M RAMSEY (1998) Floral display, pollinator visitation and reproductive success in the dioecious perennial herb Wurmbea dioica (Liliaceae). Oecologia 115: 93-101.

WASER NM (1998) Pollination, angiosperm speciation, and the nature of species boundaries. Oikos 81: 198201.

WASER NM, L CHITTKA, MV PRICE, NM WILLIAMS \& J OLLERTON (1996) Generalization in pollination systems, and why it matters. Ecology 77: 1043-1060.

WASER NM \& MV PRICE (1990) Pollination efficiency and effectiveness of bumble bees and hummingbirds visiting Delphinium nelsonii. Collectanea Botanica 19: 9-20.

WIDÉN B (1991) Phenotypic selection on flowering phenology in Senecio integrifolius, a perennial herb. Oikos 61: 205-215.

WILSON P \& JD THOMSON (1996) How do flowers diverge? En: Lloyd DG \& SCH Barrett (eds) Floral biology: 88-111. Chapman and Hall, New York, New York.

ZAMORA R (1999) Conditional outcomes of interactions: the pollinator-prey conflict of an insectivorous plant. Ecology 80: 786-795.

ZAMORA R (2000) Functional equivalence in plant-animal interactions: ecological and evolutionary consequences. Oikos 88: 442-447. 